\title{
Neck Rejuvenation with Calcium Hydroxylapatite, Hyaluronic Acid Filler, and IncobotulinumtoxinA: A Case Study
}

\author{
Min-Kee Lee, MD \\ Min-Sueng Kim, MD \\ Inee Clinic, Seoul, Korea
}

Received March 20, 2020

Revised April 4, 2020

Accepted April 12, 2020

\section{Correspondence}

Min-Kee Lee

Inee Clinic, Seoul 06030, Korea

E-mail: gapba@naver.com

https://orcid.org/0000-0002-2831-3717

(C) Korean Society for Laser, Dermatology and Trichology

(c) This is an open access article distributed under the terms of the Creative Commons Attribution NonCommercial License (http://creativecommons.org/ licenses/by-nc/4.0) which permits unrestricted noncommercial use, distribution, and reproduction in any medium, provided the original work is properly cited.

\section{Background and Objectives}

Aging of the neck is characterized by skin laxity, rhytides, loss of the mandibular contour, widening of the cervicomental angle, accumulation of submental fat, and an increased prominence of platysmal bands, in addition to others, for which many treatment options are available. In the present report, we describe a 1-day combined treatment for neck rejuvenation that addresses these signs of aging without the need for radiofrequency, highintensity focused ultrasound, laser, or other devices.

\section{Materials and Methods}

To improve skin laxity, calcium hydroxylapatite in a 1:1 dilution with normal saline or lidocaine (with or without epinephrine) was applied to the neck with a 25-gauge cannula. For horizontal neck lines, hyaluronic acid filler at a total concentration of $20 \mathrm{mg} / \mathrm{ml}$ containing $0.3 \%$ lidocaine was injected subdermally along the horizontal neck wrinkles using a 33-gauge needle and a superficial serial puncture technique. To treat hyperkinetic activity of the platysma, a total dose of $60-70 \mathrm{U}$ of incobotulinumtoxinA was divided into 60-70 injections. GAIS scores and photos were assessed at 30, 90, and 180 days after treatment.

Results

A 33-year-old female patient presented with horizontal neck wrinkles and mild aging changes with minimal fat accumulation. At 6 months after treatment, she achieved a GAIS score of 2. No serious side-effects associated with the treatment were reported.

\section{Conclusion}

In this report, we found combined treatment with $\mathrm{CaHA}$, hyaluronic acid filler, and incobotulinumtoxinA to be effective at treating signs of aging of the neck. Further studies of similar treatments for neck rejuvenation may prove beneficial.

Key words

Calcium hydroxylapatite, Hyaluronic acid filler, IncobotulinumtoxinA, Neck rejuvenation 


\section{INTRODUCTION}

Aging of the neck is characterized by changes in skin laxity, the appearance of rhytides, loss of the mandibular contour, widening of the cervicomental angle, accumulation of submental fat, volume loss, and a greater prominence of the platysmal bands. Previously, the only treatment modality for lower face and neck rejuvenation was surgery [1]. To meet increasing demands for convenient, minimally invasive, and painless antiaging treatment, clinicians have introduced the use of botulinum toxin in neck rejuvenation, with high rates of patient satisfaction [2-4]. Nevertheless, various clinical aspects and anatomical characteristics of aging of the neck make it difficult to achieve satisfactory cosmetic benefits after rejuvenation procedures. Specifically, there may be varying degrees of loosening of the skin, protrusion of the submental fat pads, and appearance of horizontal neck lines and the platysmal bands [5-7]. Thus, rejuvenation treatment of the neck often requires a multimodality approach to address these multiple facets of aging. In this report, we describe the application of a combined treatment for neck rejuvenation comprising the use of diluted calcium hydroxylapatite (CaHA) to stimulate neocollagenesis for treating skin laxity, incobotulinumtoxinA as a neuromodulator to reduce hyperkinetic activity of the platysma, and low viscosity hyaluronic acid filler to diminish the appearance of horizontal neck lines.

\section{CASE REPORT}

A 33-year-old woman presented with horizontal neck wrinkles and mild aging changes with minimal fat accumulation (Fig. 1 and 2). Prior to treatment, the neck area was

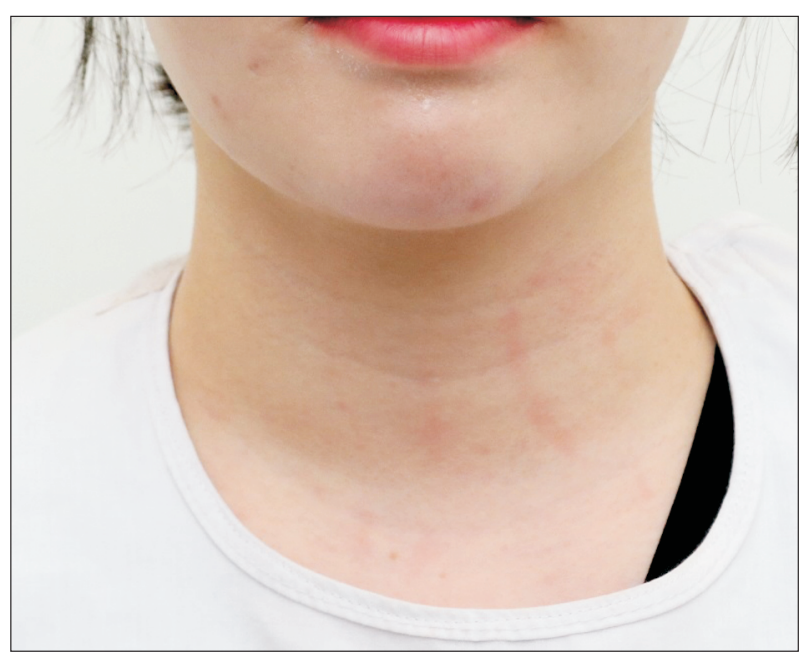

Fig. 1. Pre-treatment. first numbed with a topical anesthetic containing lidocaine and prilocaine and then cleansed with disinfectant. Afterwards, CaHA was mixed with $1.5 \mathrm{~mL}$ of $2 \%$ lidocaine (1:1 dilution), the mixing of which was repeated as many times as possible for uniform distribution of the two agents. After mixing, the diluted mixture was injected horizontally beneath the skin at the dermal-subdermal junction using a 25-gauge cannula and a fanning technique. The treated area was massaged immediately after injection to smooth out any lumps. Next, low viscosity hyaluronic acid filler at a total concentration of $20 \mathrm{mg} / \mathrm{ml}$ containing $0.3 \%$ lidocaine was injected subdermally along the deep horizontal neck wrinkles using a 33-gauge needle and a superficial serial puncture technique. The plane of injection was the deep dermis, and each injection was spaced about $0.5 \mathrm{~cm}$ apart. Manual molding was performed after the injections. Finally, for use in treating hyperkinetic activity of the platysma, one vial of $100 \mathrm{U}$ incobotulinumtoxinA was resuspended in 2.5 $\mathrm{ml}$ of normal saline. A total dose of 60-70 $\mathrm{U}$ of incobotulinumtoxinA was administered over approximately 60-70 injections: the area of the lower face and neck that benefits from incobotulinumtoxinA injections is defined as a zone bound by a line three fingerbreadths above and parallel to the lower border of the mandible, one fingerbreadth posterior to the depressor anguli oris, and all the way down over the cervicomental angle and anterior neck to the upper border of the clavicle and posteriorly to the anterior border of the sternocleidomastoid.

The patient was instructed to return for evaluation at 1 month (Fig. 3 and 4), 3 months, and 6 months (Fig. 5 and 6) after the combined procedure, at which time global aesthetic improvement scale (GAIS) scores and photos were assessed.

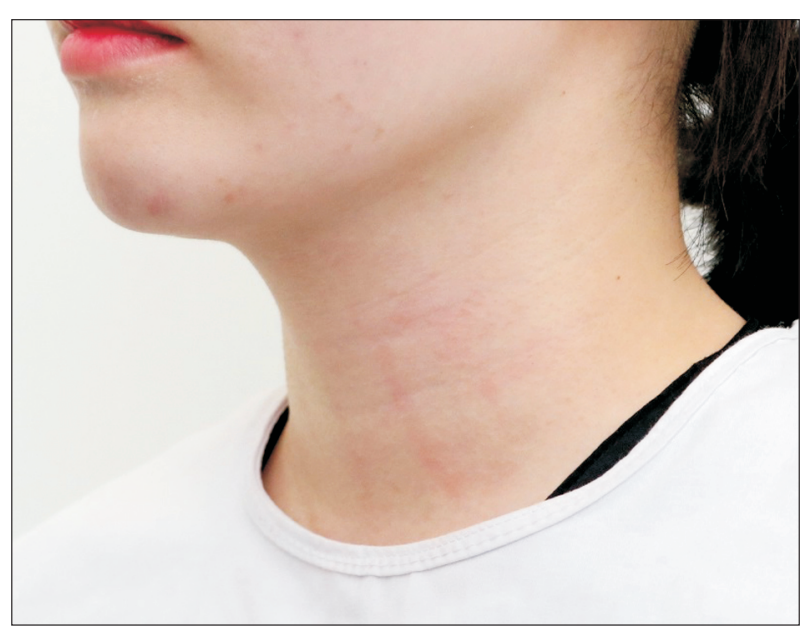

Fig. 2. Pre-treatment. 


\section{AESTHETICS}

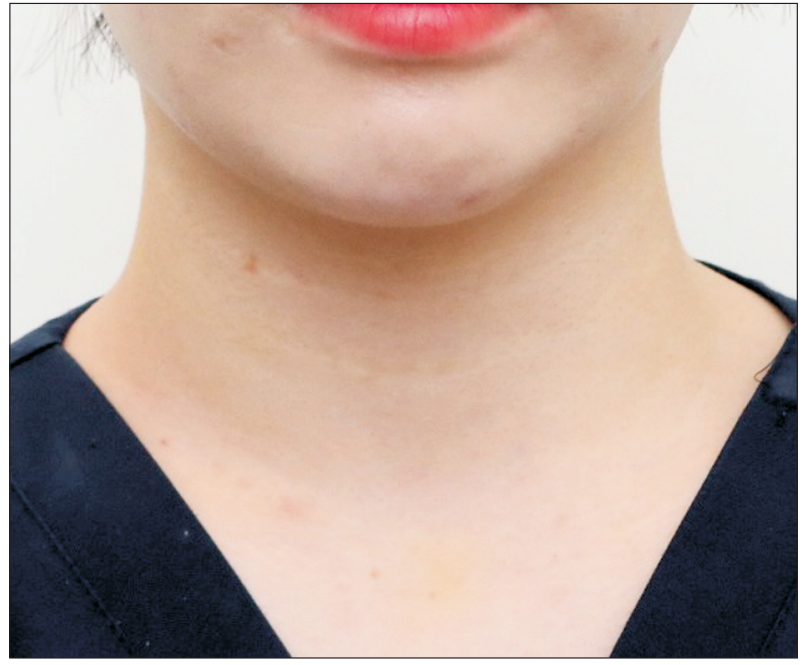

Fig. 3. At 1 month.

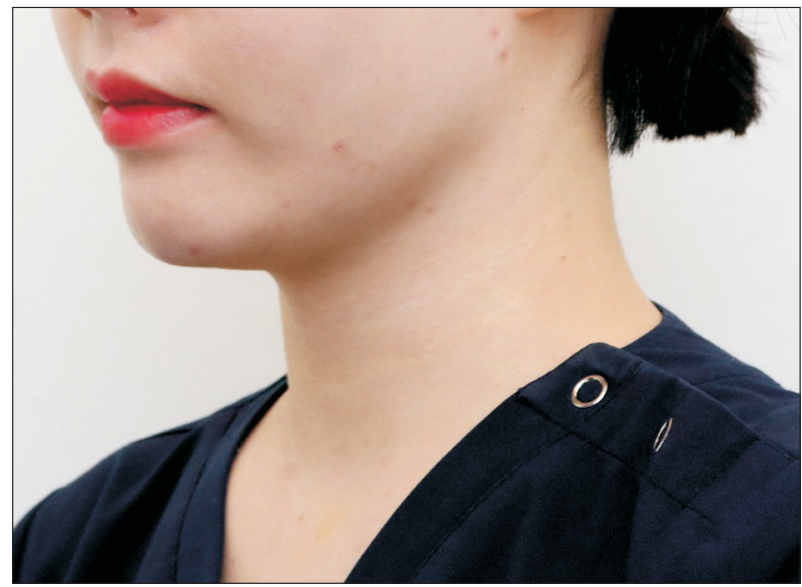

Fig. 4. At 1 month.

\section{RESULTS}

The patient achieved a final GAIS score of 2 at 6 months after combined treatment. The patient reported some bruising and lumping associated with the hyaluronic acid filler injections for up to 7 days, but none thereafter. No other side effects were recorded.

\section{DISCUSSION}

In this report, we found combined treatment with CaHA, hyaluronic acid filler, and incobotulinumtoxinA to be effective at treating signs of aging of the neck. The patient was pleased with her results at 6 months after treatment and reported no serious side-effects.

Research has shown that the collagen-stimulating properties of CaHA can be harnessed to improve the mechanical

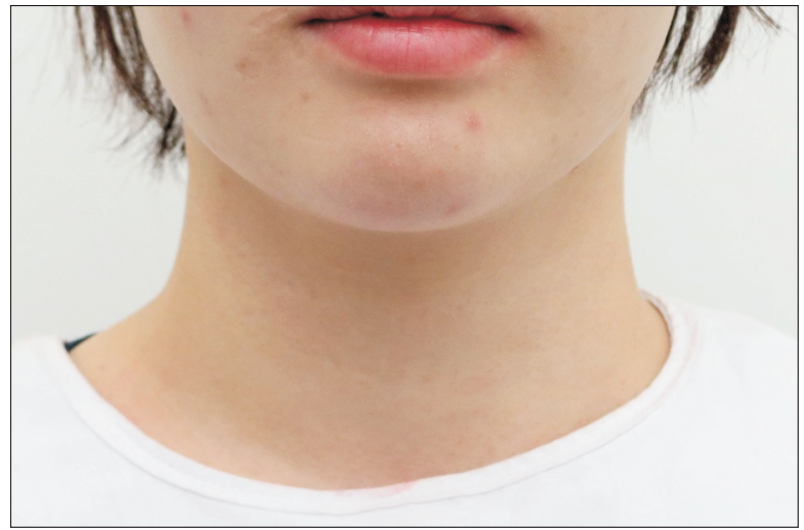

Fig. 5. At 6 months.

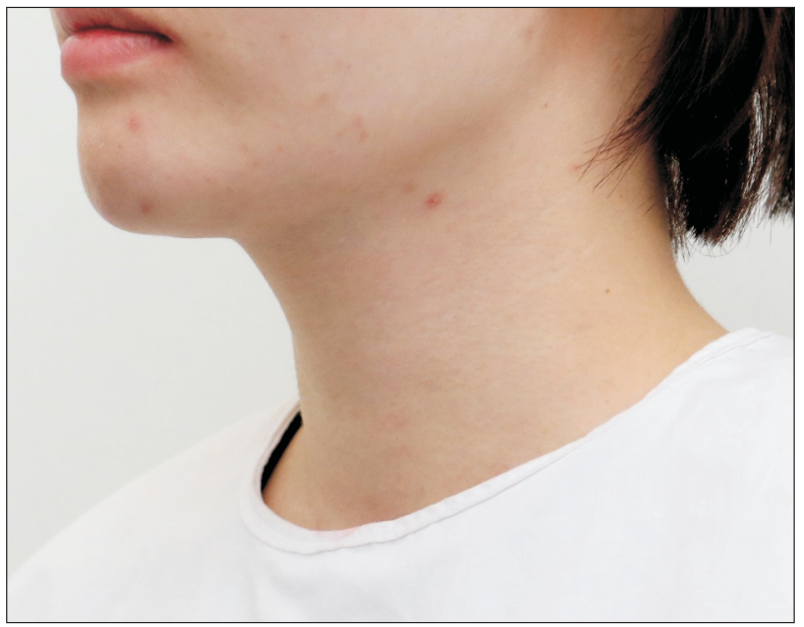

Fig. 6. At 6 months.

properties of the skin by diluting it with either lidocaine or saline $[8,9]$. The use of this technique alone to treat the skin of the neck has recently been found to stimulate the synthesis of collagen and elastin and to induce angiogenesis: treatment was associated with a significant increase in dermal thickness and improved skin mechanical properties, including elasticity and pliability [9]. In our patient, we noted that the addition of CaHA to the combined treatment helped improve the thickness of the skin, as well as its texture and elasticity, due to regenerative effects.

A characteristic feature of neck aging, neck wrinkles can vary in severity for each individual, becoming worse with increasing age, and numerous attempts have been made by clinicians to develop treatments with which to improve the appearance of neck wrinkles. The most common treatment to address neck wrinkles is resurfacing; however, it poses a long downtime and various side effects. Given these aspects, dermal fillers are also good options. The ideal filler would allow for dynamic restoration of the dermis structur- 
ally: moving as one with it, efficaciously expanding with it, withstanding mechanical stress, swelling minimally, and potentially stimulating collagenesis. With its space-filling, water binding, and collagenesis-stimulating properties, cohesive polydensified matrix hyaluronic acid filler appears to contribute to efficacious fine line treatment [10]. Indeed, we found that hyaluronic acid filler help reduced the signs of horizontal neck wrinkles in our patient.

Hyperkinetic activity and loss of tone of the platysma muscle result in vertical muscle bands and horizontal neck rhytides $[11,12]$. The platysma muscle stretches the skin of the neck downward and laterally, and in synergy with the depressor anguli oris, pulls down the lateral corners of the mouth. The muscle extends from the sternoclavicular joint, clavicular bone, and scapular acromion inferiorly to join the superficial musculoaponeurotic system of the face superiorly [13]. As they gradually detach from deep planes and become attenuated, the muscle fibers contribute to the appearance of aging of the neck [2]. Treatment with botulinum toxin injections in this area, so called "the Nefertiti lift," is a safe, standardized, and simple nonsurgical option for treating aging of the neck and lower face [14]. These intradermal injections have the desired effect of smoothening and tightening the skin due to neurochemically induced bulk atrophy of the sweat and sebaceous glands [15-19]; they also decrease the subtle pulling and tethering effects of the facial muscles where they attach to the skin. Moreover, weakening the superficial fibers of the platysma muscle with microdroplet injections decreases the appearance of horizontal neck lines and vertical bands, while the deeper muscle fibers continue to function and exert their muscle pull inward and upward, creating a more snug apposition of the platysma to the underlying neck structures. This creates a more-defined cervicomental angle and even the illusion of lifting of the jowls and jawline in cases of prominent vertical platysmal bands. In addition to improvements in horizontal lines and vertical banding, microbotox treatment of the lower face and neck has also been found to benefit the texture, color, and sheen of the neck skin [20].

The combined treatment we applied in this report did not require the use of specialized devices, such as radiofrequency, high-intensity focused ultrasound, laser, or other devices. Thus, it could be applied by clinicians with limited access thereto. Nonetheless, treatment with high-intensity focused ultrasound or other devices in addition to the diluted CaHA used in this report could potentially help further stimulate collagen or elastin production.

In conclusion, we found combined treatment with $\mathrm{CaHA}$, hyaluronic acid filler, and incobotulinumtoxinA to be effective at treating signs of aging of the neck. Further studies of similar treatments may prove beneficial to advancing 1-day treatments for neck rejuvenation and to helping clinicians choose an optimal treatment for each individual. Additionally, incorporating the use of laser, radiofrequency, and ultrasound devices may promote better results from our combined treatment and may prove to reduce the side effects associated with these devices.

\section{CONFLICT OF INTEREST}

No potential conflict of interest relevant to this article was reported.

\section{REFERENCES}

1. Daher JC. Closed platysmotomy: a new procedure for the treatment of platysma bands without skin dissection. Aesthetic Plast Surg 2011;35:866-77.

2. Matarasso A, Matarasso SL. Botulinum A exotoxin for the management of platysma bands. Plast Reconstr Surg 2003;112(5 Suppl):138S-40S.

3. Spósito MM. New indications for botulinum toxin type $A$ in treating facial wrinkles of the mouth and neck. Aesthetic Plast Surg 2002;26:89-98.

4. Kane MA. Nonsurgical treatment of platysma bands with injection of botulinum toxin a revisited. Plast Reconstr Surg 2003;112(5 Suppl):125S-26S.

5. Brandt FS, Bellman B. Cosmetic use of botulinum A exotoxin for the aging neck. Dermatol Surg 1998;24:1232-4.

6. Dedo DD. "How I do it"-plastic surgery. Practical suggestions on facial plastic surgery. A preoperative classification of the neck for cervicofacial rhytidectomy. Laryngoscope 1980;90(11 Pt 1):1894-6.

7. Dibernardo BE. The aging neck: a diagnostic approach to surgical and nonsurgical options. J Cosmet Laser Ther 2013;15:56-64.

8. Yutskovskaya Y, Kogan E, Leshunov E. A randomized, splitface, histomorphologic study comparing a volumetric calcium hydroxylapatite and a hyaluronic acid-based dermal filler. J Drugs Dermatol 2014;13:1047-52.

9. Yutskovskaya YA, Kogan EA. Improved neocollagenesis and skin mechanical properties after injection of diluted calcium hydroxylapatite in the neck and décolletage: a pilot study. J Drugs Dermatol 2017;16:68-74.

10. Sundaram H, Fagien S. Cohesive polydensified matrix hyaluronic acid for fine lines. Plast Reconstr Surg 2015;136(5 Suppl):149S-63S.

11. Knize DM. Limited incision submental lipectomy and platysmaplasty. Plast Reconstr Surg 1998;101:473-81.

12. Ellenbogen R, Karlin JV. Visual criteria for success in restoring 
the youthful neck. Plast Reconstr Surg 1980;66:826-37.

13. Tamura BM. The effect of botulinum toxin on the platysma muscle. Curr Dermatol Rep 2012;1:89-95.

14. Rose AE, Goldberg DJ. Safety and efficacy of intradermal injection of botulinum toxin for the treatment of oily skin. Dermatol Surg 2013;39(3 Pt 1):443-8.

15. Bushara KO, Park DM, Jones JC, Schutta HS. Botulinum toxin: a possible new treatment for axillary hyperhidrosis. Clin Exp Dermatol 1996;21:276-8.

16. Glogau RG. Botulinum A neurotoxin for axillary hyperhidrosis. No sweat Botox. Dermatol Surg 1998;24:817-9.

17. Naumann M, Lowe NJ. Botulinum toxin type A in treatment of bilateral primary axillary hyperhidrosis: randomised, parallel group, double blind, placebo controlled trial. BMJ 2001;323:596-9.

18. Shah AR. Use of intradermal botulinum toxin to reduce sebum production and facial pore size. J Drugs Dermatol 2008;7:847-50.

19. Jabbour SF, Kechichian EG, Awaida CJ, Tomb RR, Nasr MW. Botulinum toxin for neck rejuvenation: assessing efficacy and redefining patient selection. Plast Reconstr Surg 2017;140:9e-17e.

20. Wu WT. Microbotox of the lower face and neck: evolution of a personal technique and its clinical effects. Plast Reconstr Surg 2015;136(5 Suppl):92S-100S. 